\title{
How to Interpret Gastric Emptying Scintigraphy
}

\author{
Ju Won Seok \\ Department of Nuclear Medicine, College of Medicine, Chung-Ang University, Seoul, Korea
}

Gastric emptying scintigraphy has long been the standard method for measuring gastric motility. Various methodologies for this study have been used including meal composition, patient positioning, instrumentation, frequency of data acquisition, study length and quantitative method. For accurate quantification, it is not important which method is in use, except that the methodology should always be the same and normal values need to be derived and validated for that methodology.

(J Neurogastroenterol Motil 2011;17:189-191)

\section{Key Words}

Gastric emptying; Methods; Stomach

\section{Introduction}

Scintigraphic analysis of gastric motility is noninvasive, reproducible, simple to perform, accurate and quantitative. Ever since the introduction of more than 30 years ago, gastric emptying scintigraphy has been significantly refined and optimized over the years. Radionuclide gastric emptying studies are now well established as the standard method to evaluate gastric emptying.

\section{Standard Gastric Emptying Procedure}

Various methodologies have been used., Meal composition, patient positioning, instrumentation, frequency of data acquisition, study length and quantitative methods all vary between institutions. In general, the patient is asked to fast overnight or for at least 4 hours prior to the study. Diabetic patients should be studied in the morning, 20-30 minutes after their normal insulin dosage. A wide variety of drugs can affect gastric emptying and therefore most medication should be discontinued prior to a scintigraphic evaluation. Smoking delays gastric emptying and should be avoided.

To accurately quantify solid emptying, the radiotracer must be tightly bound to the solid meal. There is currently no consensus on the optimal test meal to study gastric emptying. Generally, radiolabel egg albumen with Tc-99m sulfur colloid is used in most nuclear medicine laboratories. The eggs are mixed with $37 \mathrm{MBq}$ Tc-99m sulfur colloid by frying or scrambling and the prepared egg product is then administered orally, often with toasted bread as an egg sandwich. Labeling efficiency is approximately $85 \%$.

Measuring simultaneous solid and liquid emptying is feasible using a dual isotope study by radiolabeling a liquid phase with In-111 DTPA (Indium-111 diethylenetriamine pentaacetic acid). A clear liquid study may be useful in a patient intolerant of

Received: February 16, 2011 Revised: February 21, 2011 Accepted: February 23, 2011

(c) This is an Open Access article distributed under the terms of the Creative Commons Attribution Non-Commercial License (http://creativecommons. org/licenses/by-nc/3.0) which permits unrestricted non-commercial use, distribution, and reproduction in any medium, provided the original work is properly cited.

*Correspondence: Ju Won Seok, MD

Department of Nuclear Medicine, College of Medicine, Chung-Ang University, 224-1 Heukseok-dong, Dongjak-Gu, Seoul 156-755, Korea

Tel: +82-2-6299-2896, Fax: +82-2-6299-2899, E-mail: ethmoid@hanmail.net

Financial support: None.

Conflicts of interest: None. 


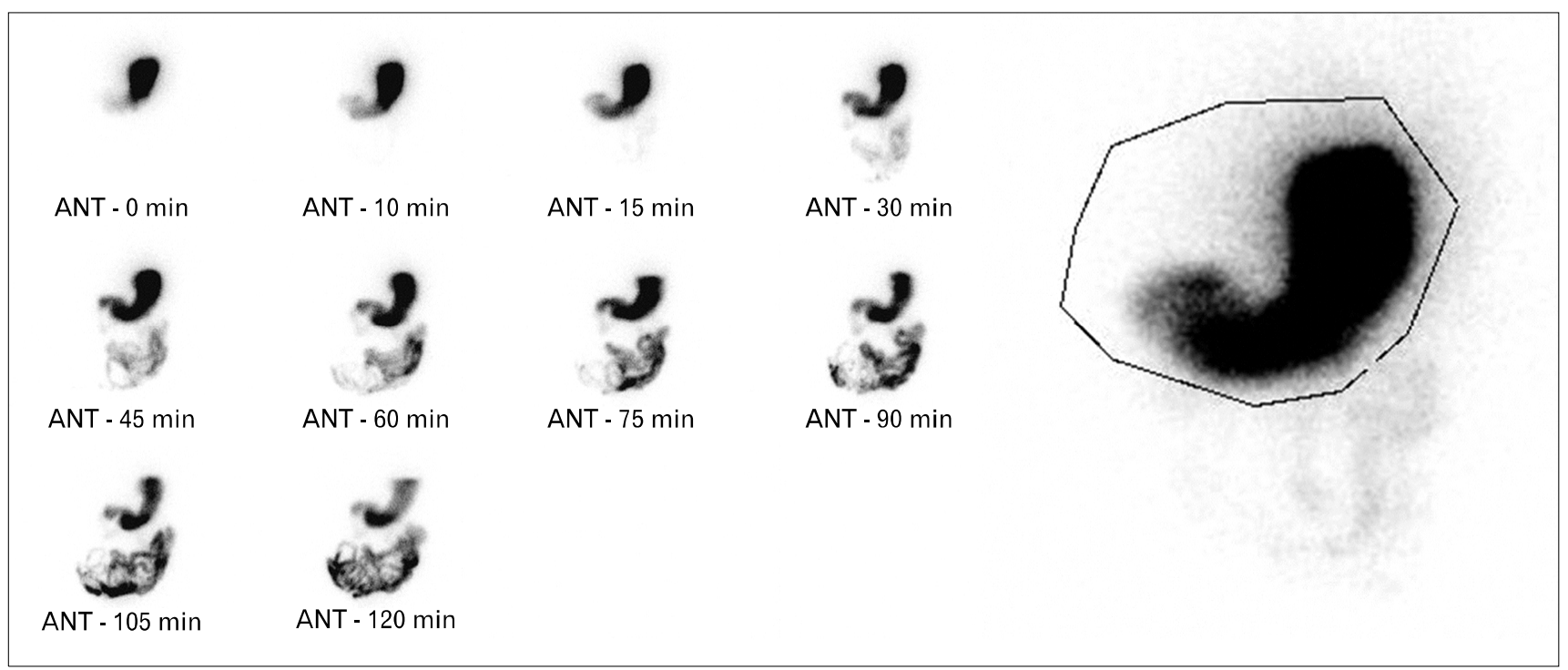

Figure 1. Data acquisition was performed for 120 minutes with intermittent imaging. The region of interest (ROI) is drawn around the activity in the entire stomach in anterior and posterior views (or the left anterior oblique view). The ROI should include any visualized activity in the fundic and antral regions of the stomach, with care to adjust the ROI to avoid activity from adjacent small bowel as in right large figure.

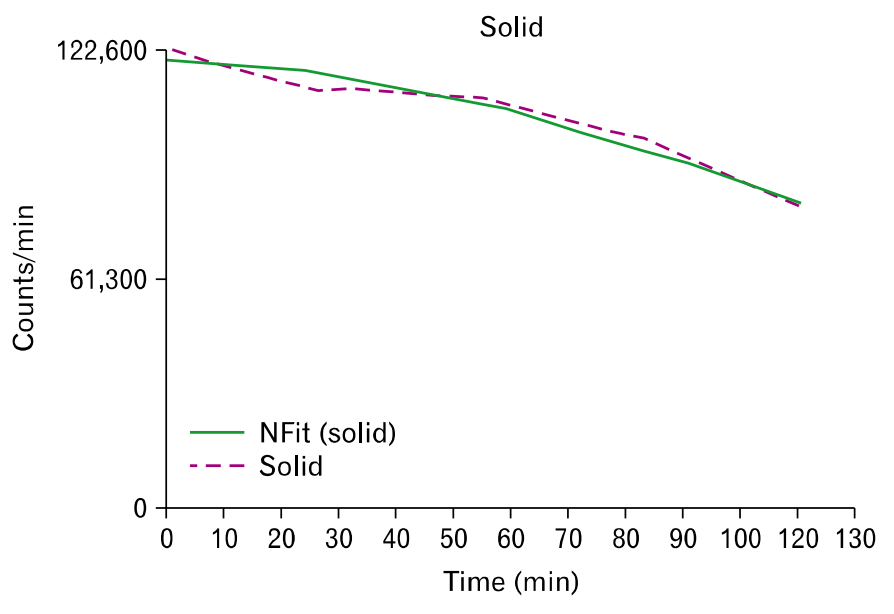

30 min after count $\%=94.9 \%$

$60 \mathrm{~min}$ after count $\%=88.2 \%$

90 min after count $\%=78.6 \%$

$120 \mathrm{~min}$ after count $\%=67.3 \%$
Gaussian fit

T MAX: $\quad 0.0 \mathrm{~min}$

T 1/2: $\quad 163.3 \mathrm{~min}$

$T$ MAX $\rightarrow$ 1/2: $\quad 163.3 \mathrm{~min}$

Cleared at $120 \mathrm{~min}: 32.7 \%$

Goodness of fit: $R=0.958$

RMS error $=2,854$

Decay corrected (Tc-99 m).

Figure 2. Image acquisition was performed at every 30 minutes for 2 hours. Remnant activity at 1 and 2 hours were $88.2 \%$ and $67.3 \%$, respectively. The half emptying time $\left(\mathrm{T}_{1 / 2}\right)$ was 163.3 minutes. Gastric emptying time was markedly delayed compared to normal value. solids but able to retain liquids. ${ }^{3,4}$ To evaluate liquid emptying, water is labeled with $3.7 \mathrm{MBq}$ In-111 DTPA. The test meal should be ingested within 5-10 minutes, after which imaging commences.

\section{Data Acquisition and Analysis}

Immediately after the completion of the meal, the patient is positioned in front of the camera. The study can be acquired on standing, sitting and supine. For accurate quantification, it is not important which method is used, except that the methodology should always be the same and normal values need to be derived and validated for that methodology.

The stomach lies obliquely within the abdomen and as food moves from the relatively posterior fundus to the relatively anterior antrum, there is an apparent increase in anterior counts due to lesser depth. For accurate quantitation, images in both anterior and posterior projections are obtained with averaging while at- 
tenuation correction should be performed routinely. The standard method for attenuation correction is the geometric mean method (square root of the product of anterior and posterior views).

Other work suggests that the use of a single left anterior oblique projection also minimizes geometric effects. ${ }^{5}$ In that projection, the stomach contents move roughly parallel to the head of gamma camera, thus minimizing the effect of attenuation. The left anterior oblique method requires no mathematical correction of attenuation.

Data acquisition is performed for 60-120 minutes either as continuous or intermittent imaging (Fig. 1). In cases where there is prolonged retention of material within the stomach, even more delayed images up to 3 hours may be required. Measurement of the half emptying time, or time required by the stomach to empty $50 \%$ of the ingested meal, is the simplest way to assess gastric transit. It is routinely and commonly used for clinical evaluation.

Other protocols call for less frequent image acquisition at every 20-30 minutes for 2-3 hours (Fig. 2). In the some study using this acquisition protocol, the normal values were $10 \%, 65 \%$ and $90 \%$, at 1, 2 and 4 hours, respectively. ${ }^{6}$ Some data suggest that the longer imaging period of 4 hours provides a higher sensitivity for detection of abnormal emptying, compared to 2 hours. ${ }^{7}$

\section{Conclusion}

Gastric emptying scintigraphy is the only satisfactory method of quantitatively measuring the rate of gastric emptying. But this method has not been standardized unfortunately and many variations exist. It is very important to establish normal ranges for the technique employed in each individual laboratory, as the results are highly dependent on the acquisition of parameters and the test meal used.

\section{References}

1. Donohoe KJ, Maurer AH, Ziessman HA, et al. Procedure guideline for adult solid-meal gastric-emptying study 3.0. J Nucl Med Technol 2009;37:196-200.

2. Maurer A. Consensus report on gastric emptying: what's needed to prevent tarnishing a gold standard? J Nucl Med 2008;49:339.

3. Lin E, Connolly LP, Drubach L, et al. Effect of early emptying on quantitation and interpretation of liquid gastric emptying studies of infants and young children. J Nucl Med 2000;41:596-599.

4. Ziessman HA, Chander A, Clarke JO, Ramos A, Wahl RL. The added diagnostic value of liquid gastric emptying compared with solid emptying alone. J Nucl Med 2009;50:726-731.

5. Ford PV, Kennedy RL, Vogel JM. Comparison of left anterior oblique, anterior and geometric mean methods for determining gastric emptying times. J Nucl Med 1992;33:127-130.

6. Tougas G, Eaker EY, Abell TL, et al. Assessment of gastric emptying using a low fat meal: establishment of international control values. Am J Gastroenterol 2000;95:1456-1462.

7. Ziessman HA, Bonta DV, Goetze S, Ravich WJ. Experience with a simplified standardized 4-hour gastric-emptying protocol. J Nucl Med 2007;48:568-572. 